\title{
Single-Unit Response of Noradrenergic Neurons in the Locus Coeruleus of Freely Moving Cats. II. Adaptation to Chronically Presented Stressful Stimuli
}

\author{
Elizabeth D. Abercrombie and Barry L. Jacobs \\ Program in Neuroscience, Department of Psychology, Princeton University, Princeton, New Jersey 08544
}

\begin{abstract}
The preceding paper described a stress-related tonic activation of noradrenergic neurons of the locus coeruleus (LC) of behaving cats. The present study examined the degree to which adaptation to stress was reflected in LC unit activity. Adaptation was defined as a reduction in the magnitude of the physiological stress response of the animal, as reflected in plasma norepinephrine level and heart rate. Adaptation of the physiological stress response occurred after 5 repeated hourly presentations of $100 \mathrm{~dB}$ white noise and after 2 hr of restraint. When these stimuli ceased to elicit significant sympathoadrenal activation, they likewise ceased to elicit activation of LC single-unit activity. These results provide further support for the hypothesis that tonic elevations in LC neuronal activity are stress related and that the LC Is involved in the CNS response to challenges to the organism.
\end{abstract}

In the preceding paper (Abercrombie and Jacobs, 1987) a sustained stress-specific increase in the single-unit activity of locus coeruleus (LC) neurons was demonstrated. The present experiment was designed to examine the responses of these neurons during adaptation to stress. It is well known that the stress response is not immutable (see below). A stimulus that may be stressful at one time may not bc at another-adaptation may occur so that the physiological response to the stimulus is reduced or eliminated. The prediction of the present experiment was that the responses of LC neurons to stressful stimuli should display a temporally parallel adaptation to that occurring with repeated or prolonged stress.

Adaptation, in the present studies, is defined as a significant reduction in the magnitude of the stress response of the animal, possibly returning to basal levels. The stress response was measured, as in the preceding paper, by changes in plasma norepinephrine (NE) and heart rate.

Adaptation to repeated stressors has been shown to be manifested as changes in both peripheral and central NE release. For example, rats exposed to repeated immobilizations show a smaller plasma NE response to subsequent immobilization than nonadapted rats exposed to this stimulus for the first time (Kvetnansky et al., 1979). In a study employing foot-shock stress (Stone and McCarty, 1983), it was found that rats subjected to

\footnotetext{
Received Dec. 9, 1986; revised Mar. 23, 1987; accepted Mar. 24, 1987.

This work was supported by NIMH Predoctoral Fellowship Grant MH09256 (to E.D.A.) and NIMH Grant MH23433 and Air Force Grant AFOSR85-0034 (to B.L.J.).

Correspondence should be addressed to Dr. Abercrombie, Department of Behavioral Neuroscience, University of Pittsburgh, Pittsburgh, PA 15260.

Copyright (C) 1987 Society for Neuroscience 0270-6474/87/092844-05\$02.00/0
}

chronic foot-shock did not show the augmented stress-induced release of central NE seen after acute foot-shock.

In a systematic study of the physiological and biochemical concomitants of restraint stress in rats, Keim and Sigg (1976) reported that signs of adaptation appeared when rats were restrained for increasing periods of time. Adrenal NE showed a peak increase after $30 \mathrm{~min}$ of restraint and returned to near control values by $2 \mathrm{hr}$. NE content in the brain was also altered over the course of prolonged restraint. While brain-stem NF. remained unchanged, hypothalamic NE decreased, gradually returning to control level. These data, as well as those discussed above, suggest that adaptation to these stressors, and possibly to others, is correlated with a reduction in the size of both the peripheral and central NE response to stress. The present experiment directly examines this issue at the single-unit level.

To our knowledge, adaptation to stressors has never been examined in the conscious cat. For this reason, plasma NE and heart rate responses to 5 hourly repeated presentations of 15 min of $100 \mathrm{~dB}$ white noise and to continuous restraint for $2 \mathrm{hr}$ were examined. This was done to document adaptation of the stress response under these conditions in behaving cats.

\section{Materials and Methods}

The general methods for the present experiment were identical to those described in the preceding paper (Abercrombie and Jacobs, 1987). Stressor methodology and data analyses differed in certain time parameters, as described below.

Repeated white noise. Cats were exposed to 15 min of $100 \mathrm{~dB}$ white noise once per hour for a total of 5 presentations. Unit activity and heart rate were recorded continuously during these periods, as well as for $15 \mathrm{~min}$ after each noise offset. Plasma NE levels were examined at $15 \mathrm{~min}$ before, minutes 1 and 15 during, and $15 \mathrm{~min}$ after noise for the first presentation of the white noise. During the fifth presentation of the white noise, plasma NE was determined for minutes 1 and 15 of the stimulus.

Prolonged restraint. Restraint was maintained for $2 \mathrm{hr}$, during which both unit activity and heart rate were recorded continuously. The recording session continued for $15 \mathrm{~min}$ following the termination of restraint. Plasma samples obtained 15 min before, 1, 15, and $120 \mathrm{~min}$ during, and $15 \mathrm{~min}$ after restraint were analyzed.

Data analysis. Plasma NE values were analyzed using ANOVA with repeated measures and post hoc tests to evaluate the significance of difference from baseline. The heart rate values obtained in parallel with the plasma samples were analyzed in an identical manner. The Pearson correlation coefficient was determined for the plasma NE and heart rate response of individual animals for each manipulation.

The unit data for adaptation to white noise were analyzed by calculating the mean discharge rate of the cells studied during minutes 1,5 , 10 , and 15 , as well as $15 \mathrm{~min}$ after each consecutive presentation. These mean discharge rates were obtained from 6 consecutive $10 \mathrm{sec}$ time samples and expressed as spikes/sec \pm SEM. The unit responses to the first presentation and the fifth presentation of the noise were analyzed 
REPEATED WHITE NOISE

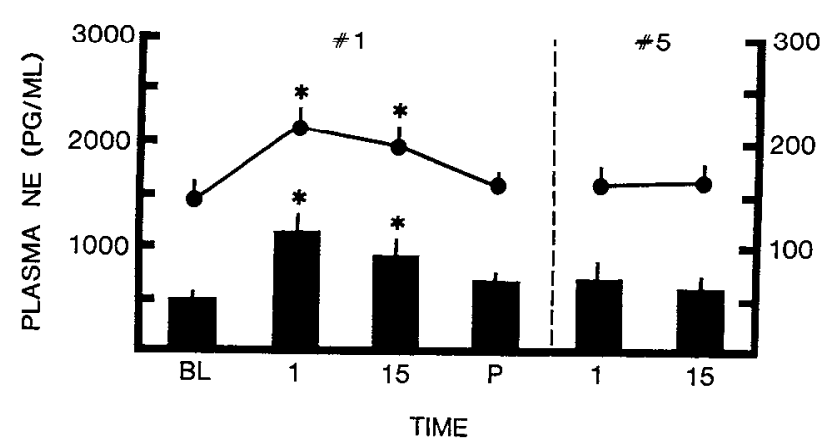

Figure 1. Plasma NE (solid bars) and accompanying heart rate (circles) responses to presentation \#1 (left) and presentation \#5 (right) of white noise $(n=7)$. Data shown are means \pm SEM. $*$, significantly greater than baseline $(p<0.05)$. $B L$, baseline; 1 , minute 1 of manipulation; 15 , minute 15 of manipulation; $P, 15$ min postmanipulation.

using one-way ANOVA with repeated measures. Post-hoc tests were used to compare firing rates within presentations 1 and 5 to the active waking (AW) baseline firing level. Presentations 1 and 5 were chosen for analysis a priori since the time course of adaptation to this stimulus could not be predicted beforehand. The overall effect of repeated exposure to the white noise on LC unit activity was assessed with a 2-way (presentation $\times$ time) repeated measures ANOVA. Duncan's post hoc test was employed to examine differences between presentations.

The unit responses to $2 \mathrm{hr}$ restraint were determined for minutes 1 , $5,10,15,30,60,90$, and 120 , as well as 15 min after stimulus (obtained from 6 consecutive $10 \mathrm{sec}$ time samples as spikes/sec $\pm \mathrm{SEM}$ ). These values were then analyzed using one-way ANOVA with repeated measures and post hoc tests to assess the significance of difference from AW baseline.

The heart rate changes accompanying the unit changes in response to repeated whitc noise and prolonged restraint were analyzed using statistical tests identical to those described above.

\section{Results}

\section{Plasma NE and accompanying heart rate responses}

Repeated white noise. The effects of 5 repeated hourly presentations of 15 min of $100 \mathrm{~dB}$ white noise on plasma NE levels and heart rate were examined in 7 cats (Fig. 1). The data for the first white noise presentation are those of the preceding paper (Abercrombie and Jacobs, 1987). Plasma NE was significantly increased over baseline at minutes $1(146 \%)$ and $15(100 \%)$, whereas by $15 \mathrm{~min}$ poststimulus it was no longer significantly elevated. The data from minutes 1 and 15 of the fifth white noise presentation showed that, although plasma NE was slightly incrcased at these time points (51 and $34 \%$, respectively), the levels were not significantly greater than baseline.

Parallel changes in heart rate were obtained in response to repeated white noise. Significant elevations in heart rate were obtained during minutes 1 and 15 of the first stimulus presentation. Heart rate showed significant elevations from a baseline level of $143 \pm 9$ beats/min to $213 \pm 13$ and $198 \pm 15$ beats/ min at minutes 1 and 15 , respectively. By the fifth presentation, however, the stimulus no longer elicited a significant heart rate increase at either minute 1 or 15 , although a modest rise was observed, i.e., $161 \pm 10$ beats/min at minute 1 and $164 \pm 10$ beats/min at minute 15 .

Prolonged restraint. Plasma NE levels and heart rate were studied in 7 cats over the course of $2 \mathrm{hr}$ of restraint (Fig. 2). In agreement with results reported in the preceding paper (Abercrombie and Jacobs, 1987), plasma NE showed a significant
PROLONGED RESTRAINT

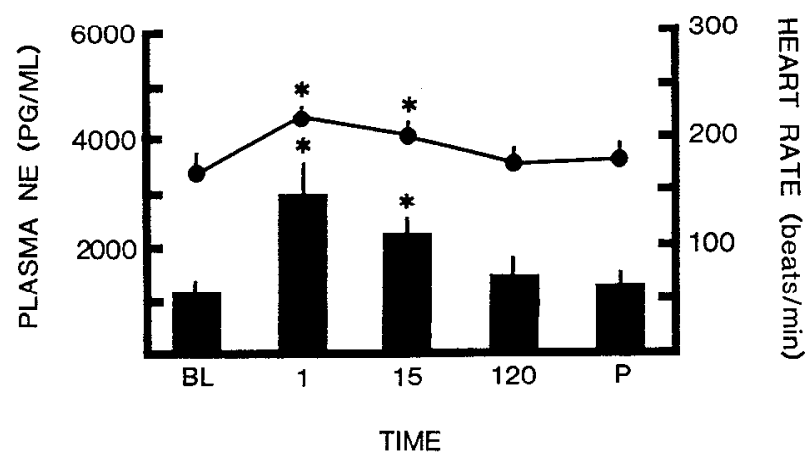

Figure 2. Plasma NE (solid bars) and accompanying heart rate (circles) responses to prolonged restraint $(n=7)$. Data shown are means \pm SEM. $*$, significantly greater than baseline $(p<0.05)$. $B L$, baseline; 1 , minute 1 of manipulation; 15 , minute 15 of manipulation; 120 , minute 120 of manipulation; $P, 15 \mathrm{~min}$ postmanipulation.

increase over baseline levels at minutes $1(222 \%)$ and $15(150 \%)$ of the stimulus. By minute 120 , however, the plasma NE level was no longer significantly different from baseline $(27 \%$ over baseline). By 15 min after restraint, NE levels were also not significantly different from baseline ( $10 \%$ over baseline).

The changes in heart rate in response to $2 \mathrm{hr}$ of restraint were quite similar to the plasma NE changes. That is, heart rate was significantly elevated over baseline $(160 \pm 15$ beats $/ \mathrm{min})$ at minutes $1(216 \pm 8$ beats $/ \mathrm{min})$ and $15(197 \pm 3$ beats $/ \mathrm{min})$ but was no longer significantly increased at minute $120(177 \pm 13$ beats/min). Heart rate 15 min after restraint $(172 \pm 13$ beats/ $\mathrm{min}$ ) was likewise not significantly different from baseline.

The Pearson correlation coefficients obtained for the plasma $\mathrm{NE}$ and heart rate responses of each animal for these manipulations showed these 2 variables to be highly correlated. The means of these values were $r=0.87$ for repeated white noise and $r=0.86$ for prolonged restraint.

\section{$L C$ unit activity and accompanying heart rate responses}

Repeated white noise. The LC NE unit response to 5 repeated hourly presentations of $15 \mathrm{~min}$ of $100 \mathrm{~dB}$ white noise was studied in 6 cells along with the concomitant effects on heart rate (Fig. 3). In response to the first presentation of the noise, unit activity showed a highly significant increase $[F(5,25)=8.44$, $p=0.0001]$. The increase was significantly greater than baseline at each time point examined during the stimulus. Mean unit activity was $2.0 \pm 0.3 \mathrm{spikes} / \mathrm{sec}$ during the first minule compared with $0.8 \pm 0.2$ spikes/sec during AW. Unit activity was no longer significantly different from baseline at $15 \mathrm{~min}$ after noise offset $(0.7 \pm 0.2 \mathrm{spikes} / \mathrm{sec})$. By the fifth presentation of the white noise, no significant increases in mean unit activity were elicited despite an overall effect $[F(5,25)=5.15, p=0.03]$. The significance of the overall effect was due to the fact that the level of activity $15 \mathrm{~min}$ poststimulus $(0.4 \pm 0.1 \mathrm{spikes} / \mathrm{sec})$ was significantly lower than AW baseline. The highest rate of unit activity obtained during the fifth presentation was $0.8 \pm 0.1$ spikes/sec during minute 1 .

Much the same pattern was observed for the heart rate responses recorded simultaneously. That is, in response to the first presentation of the white noise, heart rate increased significantly $[F(5,25)=25.82, p<0.0001]$. At minute 1 , heart rate was $190 \pm 6$ beats/min compared with a baseline level of $154 \pm 3$ 

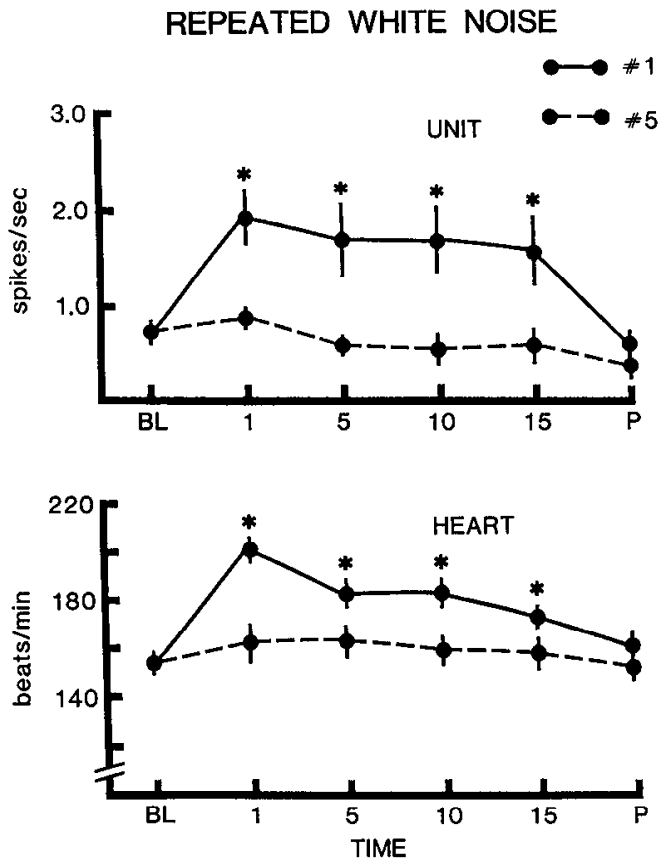

Figure 3. Comparison of LC NE unit (top) and accompanying heart rate (bottom) responses to presentation \#1 (solid lines) and presentation \#5 (broken lines) of white noise $(n=6)$. Data shown are means \pm SEM. $*$, significantly greater than baseline $(p<0.05) . B L$, baseline; 1 , minute 1 of manipulation; 5 , minute 5 of manipulation; 10 , minute 10 of manipulation; 15 , minute 15 of manipulation; $P, 15$ min postmanipulation.

beats $/ \mathrm{min}$. This increase was evident over the entire $15 \mathrm{~min}$ period. Heart rate returned to baseline levels by $15 \mathrm{~min}$ after noise offset $(161 \pm 5$ beats $/ \mathrm{min})$. By the fifth presentation, the noise no longer elicited significant changes in heart rate $[F(5,25)-1.14, p-0.37]$. The mean heart rate during minute 1 was $161 \pm 7$ beats $/ \mathrm{min}$.

An ANOVA examining the NE unit responses over time to each of the 5 presentations of the white noise revealed a significant interaction of presentation and time $[F(4,25)=3.77$, $p=0.02]$. Post hoc tests showed that the mean levels of unit activity during minutes $1,5,10$, and 15 of the first presentation were significantly greater than those observed at the same time points during the fifth.

A significant interaction of presentation and time was also found for heart rate $[F(4,25)=2.68, p=0.05]$. The heart rate levels at minutes $1,5,10$, and 15 of the first white noise presentation were significantly greater than the corresponding heart rate levels during the fifth presentation.

Prolonged restraint. The effect of $2 \mathrm{hr}$ of restraint on $\mathrm{LC}$ unit activity was examined in 6 cells (Fig. 4); parallel changes in heart rate were also studied. The overall effect on unit activity was found to be highly significant $[F(8,40)=4.66, p=0.0004]$. Post hoc tests revealed a significant elevation in unit activity at minutes $1,5,10$, and 15 of the stimulus compared with baseline. Mean unit activity was $2.2 \pm 0.4$ spikes/sec during the first minute compared with $0.8 \pm 0.1$ spikes/sec during AW. By minute 30 , and for the remaining duration of the stressor, unit activity was no longcr significantly different from baseline levels.

An examination of the corresponding changes in heart rate produced similar results. The overall effect was significant $[F(8,40)=3.42, p=0.004]$, with post hoc tests revealing significant elevations over baseline at minutes $1,5,10,15,30$, and
PROLONGED RESTRAINT
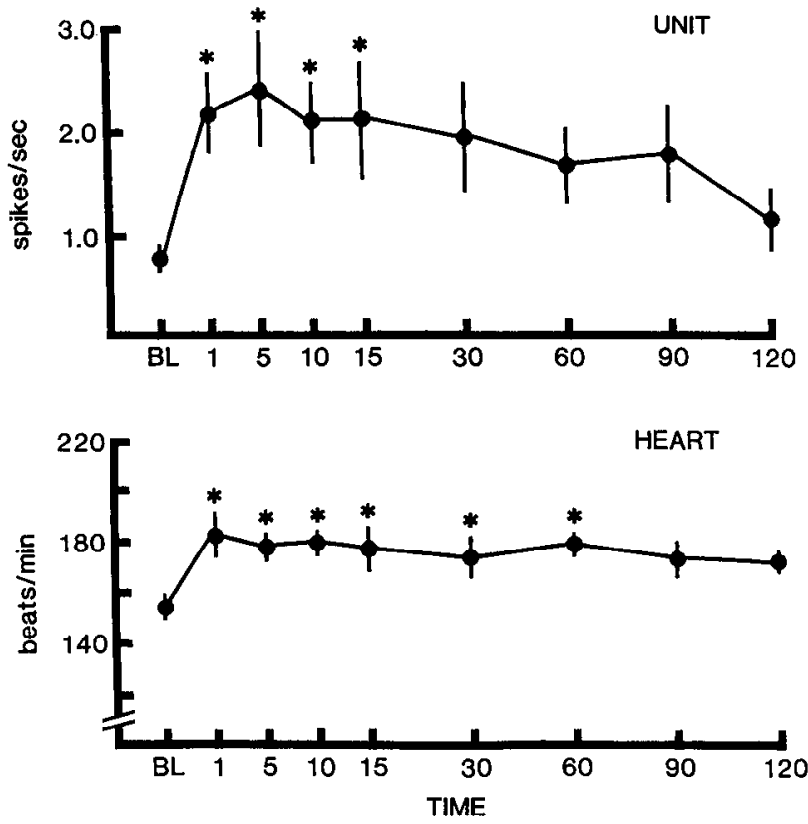

Figure 4. Summary of LC NE unit (top) and accompanying heart rate (bottom) responses to prolonged restraint $(n=6)$. Data shown are means \pm SEM. *, significantly greater than baseline $(p<0.05)$. $B L$, baseline; $I$, minute 1 of manipulation; 5 , minute 5 of manipulation; 10 , minute 10 of manipulation; 15, minute 15 of manipulation; 30 , minute 30 of manipulation; 60 , minute 60 of manipulation; 90 , minute 90 of manipulation; 120 , minute 120 of manipulation.

60. Mean heart rate was $183 \pm 9$ beats/min during minute 1 compared with a baseline of $154 \pm 5$ beats $/ \mathrm{min}$. Heart rate declined to a level not significantly different from baseline by minute 90 , remaining at that level for the duration of the stimulus.

\section{Discussion}

The first part of the present experiment examined the adaptation of the physiological stress response to repeated or prolonged stressors in behaving cats. Adaptation was defincd as a reduction in plasma NE and heart rate from significantly elevated levels to levels no longer significantly different from baseline. These parameters were examined in response to 5 repeated hourly presentations of 15 min of $100 \mathrm{~dB}$ white noise and a single 2-hrlong restraint.

We have previously shown (Abercrombie and Jacobs, 1987) that both plasma NE and heart rate were significantly elevated when sampled 1 and $15 \mathrm{~min}$ after onset of either white noise or restraint. Upon further investigation, the present study demonstrated that adaptation to both repeated white noise and prolonged restraint occurred. When white noise was presented for a fifth time, neither plasma NE nor heart rate responded with a significant increase. Similarly, when plasma NE and heart rate were examined over the course of $120 \mathrm{~min}$ restraint, the initial significant elevation in these measures was found to have adapted. Thus, the physiological response to repeated or prolonged stressors significantly diminished despite the fact that the physical nature of the stimuli remained unchanged.

The 2 variables, plasma NE level and heart rate, were highly correlated during adaptation to stress. These results provide 
further support for the use of tonic heart rate as an index of sympathoadrenal activation under the conditions of the present experiments. Thus, in those cats in which LC unit activity was studied, it was also found that white noise failed to elicit a significant increase in heart rate by the fifth presentation and that heart rate was no longer significantly elevated by $120 \mathrm{~min}$ during restraint. On the basis of these observations it is concluded that adaptation of the stress response in behaving cats occurs in response to the repeated presentation of $100 \mathrm{~dB}$ white noise or prolonged application of restraint.

Having established that adaptation to white noise and restraint did occur under the conditions of the present experiment, the response of LC units over the course of adaptation was examined. It was predicted that if the activity of LC neurons was stress related, as indicated in the preceding paper (Abercrombie and Jacobs, 1987), then the response of these neurons to stressful stimuli would adapt in parallel with the adaptation of the physiological stress response.

In response to the first presentation of the whitc noisc, LC units displayed a significant, sustained increase in firing rate that is in agreement with the results of the preceding paper (Abercrombie and Jacobs, 1987). By the fifth presentation of the noise, however, the firing rate of the LC neurons studied showed no significant response to the stimulus. This lack of response to the fifth presentation of white noise was not attributable to broadfrequency adaptation in the auditory system since a similar brief excitatory unit response to a click was obtained in these neurons during baseline and after the fifth white noise presentation (E. D. Abercrombie and B. L. Jacobs, unpublished observations).

LC unit activity showed a significant, sustained increase over the first $15 \mathrm{~min}$ of the $2 \mathrm{hr}$ restraint and then declined to levels not significantly different from baseline for the remainder of the stimulus.

Taken together with the results of the preceding paper, the present study provides additional evidence that the activity of LC neurons does indeed increase in association with sympathoadrenal activation (Elam et al., 1986; Reiner, 1986; Morilak et al., 1987a, b). In the present study, the relationship between sympathoadrenal activity and LC unit activity was extended to include adaptation to stress. When a significant sympathoadrenal activation in response to the stimuli examined was no longcr apparent, activation of LC neurons was also no longer evident.

The data of both this and the preceding paper, although supporting the idea that central and peripheral NE systems are activated in parallel, do not necessarily imply, however, that the 2 systems in question are functioning in a fixed interdependent manner. Previous studies have shown that various somatosensory and autonomic stimuli elicit a virtually parallel activation of $\mathrm{LC}$ neurons and peripheral sympathetic nerves in anesthetized rats (Elam et al., 1984, 1986). The parallelism between LC activity and sympathetic nerve activity observed in these aforementioned studies was not absolute, however. For example, the response of $\mathbf{L C}$ neurons to prolonged noxious thermal stimulation of the tail in anesthetized rats was shown to attenuate completely within a few minutes, whereas peripheral sympathetic nerve activity remained elevated throughout the stimulation. Likewise the response of $L C$ neurons in unanesthetized cats to both hypotension and hypoglycemia has been shown to diminish more rapidly than the concomitant sympathoadrenal activation (Morilak et al., 1987a, b). A somewhat similar dissociation was indicated in the present study. During the course of prolonged restraint stress, for example, the response of LC neurons appeared to adapt more quickly than the sympathoadrenal response reflected by heart rate (see Fig. 4). These results, although far from conclusive, support the idea that the central and peripheral NE systems function in a parallel, complementary fashion, rather than being inextricably linked.

The data of the present studies point to a discriminative capacity in the response of LC NE neurons to environmental stimuli. That is, a threshold seems to exist that stimuli must exceed in order to evoke more than a transient activation of the LC. Furthermore, when stimuli no longer exceed this threshold, $L C$ is no longer tonically activated. These results fit nicely with the hypothesis that $L C$ is involved in responding to stimuli of urgency to the well-being of the organism (Amaral and Sinnamon, 1977; Grant and Redmond, 1984; Aston-Jones, 1985; Elam et al., 1986; Jacobs, 1986). The ultimate consequence of this activational specificity in terms of postsynaptic action presumably would be to augment the preparedness of the organism to cope effectively with stressful conditions. The analogy to the function of the sympathetic ganglia is once again apparent.

Finally, the results of the present studies indicate that LC neurons may play a critical role in the etiology of stress-related disorders resulting from repeated or prolonged stress to which adaptation fails to, or cannot, occur. These might include, among others, hypertension, insomnia, and ulcers.

\section{Summary}

The present experiment is the first to examine the degree to which adaptation to stress is reflected in the activity of a group of brain neurons in behaving animals. We have shown that when stressful environmental stimuli lose their ability to evoke a significant sympathoadrenal activation, they likewise fail to elicit a significant activation of $\mathrm{LC}$ neurons. These data are supportive of the hypothesis that the activity of LC neurons is stress related and that the LC is involved in the CNS response to challenges to the organism.

\section{References}

Abercrombie, E. D., and B. L. Jacobs (1987) Single-unit response of noradrenergic neurons in the locus coeruleus of freely moving cats. I. Acutely presented stressful and nonstressful stimuli. J. Neurosci. 7: 2837-2843.

Amaral, D. G., and H. M. Sinnamon (1977) The locus coeruleus: Neurobiology of a central noradrenergic nucleus. Prog. Neurobiol. 9 $147-196$.

Aston-Jones, G. (1985) Behavioral functions of locus coeruleus derived from cellular attributes. Physiol. Psychol. 13: 118-126.

Elam, M., T. Yao, T. H. Svensson, and P. Thoren (1984) Regulation of locus coeruleus neurons and splanchnic, sympathetic nerves by cardiovascular afferents. Brain Res. 290: 281-287.

Elam, M., T. H. Svensson, and P. Thoren (1986) Locus coeruleus neurons and sympathetic nerves: Activation by cutaneous sensory afferents. Brain Res. 366: 254-261.

Grant, S. J., and D. E. Redmond, Jr. (1984) Neuronal activity of the locus ceruleus in awake Macaca arctoides. Exp. Neurol. 84: 701-708.

Jacobs, B. L. (1986) Single unit activity of locus coeruleus neurons in behaving animals. Prog. Neurobiol. 27: 183-194.

Keim, K. L., and E. B. Sigg (1976) Physiological and biochemical concomitants of restraint stress in rats. Pharmacol. Biochem. Behav. 4: 289-297.

Kvetnansky, R., R. McCarty, N. B. Thoa, C. R. Lake, and I. J. Kopin (1979) Sympatho-adrenal responses of spontaneously hypertensive rats to immobilization stress. Am. J. Physiol. 236: H457-H462.

Morilak, D. A., C. A. Fornal, and B. L. Jacobs (1987a) Effects of physiological manipulations on locus coeruleus neuronal activity in freely moving cats: II. Cardiovascular challenge. Brain Res. (in press).

Morilak, D. A., C. A. Fornal, and B. L. Jacobs (1987b) Effects of 
physiological manipulations on locus coeruleus neuronal activity in freely moving cats: III. Glucoregulatory challenge. Brain Res. (in press). Reiner, P. B. (1986) Correlational analysis of central noradrenergic neuronal activity and sympathetic tone in behaving cats. Brain Res. 378: 86-96.
Stone, E. A., and R. McCarty (1983) Adaptation to stress: Tyrosine hydroxylase activity and catecholamine release. Neurosci. Biobehav. Rev. 7: 29-34. 